OPEN ACCESS

Edited by:

Clifton D. Fuller,

University of Texas MD Anderson

Cancer Center, United States

Reviewed by:

Yuanzeng Min,

University of Science and Technology of China, China

Fiori Alite,

Geisinger Commonwealth School of Medicine, United States

*Correspondence:

Stella Mook

S.Mook-2@umcutrecht.n

Specialty section:

This article was submitted to

Radiation Oncology,

a section of the journal

Frontiers in Oncology

Received: 10 November 2020

Accepted: 02 February 2021

Published: 22 March 2021

Citation:

Lee SL, Bassetti M, Meijer GJ and Mook S (2021) Review of MR-Guided Radiotherapy for Esophageal Cancer.

Front. Oncol. 11:628009.

doi: 10.3389/fonc.2021.628009

\section{Review of MR-Guided Radiotherapy for Esophageal Cancer}

\author{
Sangjune Laurence Lee ${ }^{1}$, Michael Bassetti ${ }^{2}$, Gert J. Meijer ${ }^{3}$ and Stella Mook ${ }^{3 *}$ \\ ${ }^{1}$ Department of Oncology, Division of Radiation Oncology, Tom Baker Cancer Centre, University of Calgary, Calgary, \\ AB, Canada, 2 Department of Human Oncology, University of Wisconsin Hospital and Clinics, Madison, WI, United States, \\ ${ }^{3}$ Department of Radiation Oncology, University Medical Center Utrecht, Utrecht University, Utrecht, Netherlands
}

In this review, we outline the potential benefits and the future role of MRI and MR-guided radiotherapy (MRgRT) in the management of esophageal cancer. Although not currently used in most clinical practice settings, MRI is a useful non-invasive imaging modality that provides excellent soft tissue contrast and the ability to visualize cancer physiology. Chemoradiation therapy with or without surgery is essential for the management of locally advanced esophageal cancer. MRI can help stage esophageal cancer, delineate the gross tumor volume (GTV), and assess the response to chemoradiotherapy. Integrated MRgRT systems can help overcome the challenge of esophageal motion due to respiratory motion by using real-time imaging and tumor tracking with respiratory gating. With daily on-table $\mathrm{MRI}$, shifts in tumor position and tumor regression can be taken into account for onlineadaptation. The combination of accurate GTV visualization, respiratory gating, and online adaptive planning, allows for tighter treatment volumes and improved sparing of the surrounding normal organs. This could lead to a reduction in radiotherapy induced cardiac toxicity, pneumonitis and post-operative complications. Tumor physiology as seen on diffusion weighted imaging or dynamic contrast enhancement can help individualize treatments based on the response to chemoradiotherapy. Patients with a complete response on $\mathrm{MRI}$ can be considered for organ preservation while patients with no response can be offered an earlier resection. In patients with a partial response to chemoradiotherapy, areas of residual cancer can be targeted for dose escalation. The tighter and more accurate targeting enabled with MRgRT may enable hypofractionated treatment schedules.

Keywords: MRI, esophageal cancer, adaptive radiotherapy, respiratory motion, cardiac toxicity

\section{INTRODUCTION}

Esophageal cancer is the seventh most common type of cancer worldwide with the sixth most common cause of cancer-related death (1). Currently, neoadjuvant chemoradiotherapy (nCRT) followed by an esophagectomy is standard of care for patients with locally advanced resectable esophageal carcinoma $(2,3)$. Definitive chemoradiotherapy is the preferred approach for unresectable locally advanced esophageal cancer or for patients who decline or are unfit for surgery $(4,5)$. Thus, radiotherapy plays an important role in the treatment of esophageal cancer. Although nCRT results in an increase in R0 resection rate, locoregional control and improved 
overall survival, 5-year overall survival remains poor after trimodality treatment. Moreover, after definitive CRT, disease persistence and locoregional recurrence are common modes of treatment failure, especially in the primary tumor region $(6,7)$. These poor outcomes warrant improvements in radiotherapy for esophageal cancer patients. This article will provide an overview of the potential benefit and future role of MRI and MR-guided radiotherapy (MRgRT) in esophageal carcinoma.

\section{THE ROLE OF MRI IN ESOPHAGEAL CANCER}

\section{Staging}

Endoscopic ultrasound (EUS), computed tomography (CT), and positron emission tomography (PET) are typically used for initial staging of esophageal cancer (8). However, all these imaging techniques have limitations with regard to accurate staging, precise tumor delineation for radiotherapy and accurate response assessment after CRT. MRI is a non-invasive technique that provides excellent soft tissue contrast and allows for imaging of cancer physiology. Using T2-weighted (T2W) and diffusion-weighted imaging (DWI), stage T1 tumors can be detected in $33 \%$ of cases, T2 in 58\%, T3 in $96 \%$ and T4 in $100 \%$. MRI has a sensitivity of $38-62 \%$ and specificity of $68-85 \%$ for $\mathrm{N}$-staging, making it a useful alternative especially in cases where the endoscope cannot pass an obstructing tumor (9). While MRI has had limited historical utilization in esophageal cancer, advances in MRI technology, including faster pulse sequences, cardiac and respiratory gating and surface coils, have improved the resolution of MRIs $(10,11)$. As these techniques continue to advance it promises greater use of MRI staging for esophageal cancer.

\section{Delineation}

Accurate tumor delineation is essential to ensure adequate target coverage while limiting dose to surrounding organs at risk (OARs). Accurate gross tumor volume (GTV) delineation is especially important when cone down or boost strategies are applied. Delineation of the GTV of locally advanced esophageal carcinoma is usually based on CT, FDG-PET, endoscopy, and EUS. Despite this multimodality approach for tumor delineation, the interobserver variability remains substantial, especially in cranial caudal direction (12). The excellent soft tissue contrast of MRI could potentially increase the accuracy of GTV delineation. The GTV appears smaller on breath hold T2W and DWI compared to conventional PET-CT which is acquired during free-breathing. Moreover, the addition of DWI to T2w MRI reduced the variability of the caudal border in tumors involving the GE-junction, showing the potential value of DWI in these cases (12). In a study of 42 patients with esophageal squamous cell carcinoma who underwent breath hold CT and DWI MRI followed by an esophagectomy, the difference in tumor length between CT and pathology was $3.6 \mathrm{~mm}$ while the difference in length between DWI and pathology was as low as $0.5 \mathrm{~mm}$ (13). Despite the excellent soft tissue contrast provided by MRI a recent study showed that MRI based target delineation did not lead to reduced interobserver variability (12). This might be due to the limited observer experience to date with contouring esophageal tumors on MRI and image acquisition characteristics (axial plane only, slice thickness of $6.5 \mathrm{~mm})$.

\section{Response Assessment}

After trimodality treatment, approximately one third of patients have a pathological complete response (pCR) (14). Patients who achieve a complete response after nCRT are likely to be unnecessarily exposed to the risks of esophagectomy, with up to $5 \%$ mortality, substantial morbidity and a substantial impact on quality of life $(14,15)$. Unfortunately, current techniques do not reliably identify complete responders (16). If these patients could be accurately identified prior to surgery, surgery might be omitted without jeopardizing outcomes.

Conversely, nearly one fifth of patients have more than $50 \%$ vital residual tumor cells in the tumor bed at histopathological examination after nCRT and are considered non-responders. These non-responders are exposed to nCRT related toxicity, probably without benefit. Therefore, accurate identification of non-responders early during the course of nCRT may allow for alternative treatment strategies, such as neoadjuvant treatment intensification, change in chemotherapy, or termination of ineffective neoadjuvant treatment and early surgery.

A meta-analysis of the current literature examining the diagnostic accuracy of clinically routine studies such as endoscopic biopsies, EUS, and PET-CT for detecting residual disease after nCRT showed that single modalities were insufficiently accurate (16). Another meta-analysis on the ability of various imaging modalities for detecting pathological complete response (pCR) showed pooled sensitivities of 0.35 , $0.62,0.01$, and 0.80 and pooled specificities of $0.83,0.73,0.99$, and 0.83 for CT, PET-CT, EUS and MRI respectively (17).

DWI and the derived apparent diffusion coefficient (ADC) and intravoxel incoherent motion (IVIM) models reflect tissue cellular density, extracellular-space tortuosity, and the integrity of cellular membranes (18). Recently, promising results for response prediction have been reported for this functional imaging modality. Baseline DWI prior to CRT therapy, interim DWI midway through treatment, and the change in between baseline and interim imaging have been found to be prognostic and predictive biomarkers (19-24). The relative change in ADC during the first 2 weeks of CRT appears to be the most predictive for residual cancer with a sensitivity of $100 \%$ and specificity of $75 \%(19,20)$.

In addition to DWI, dynamic contrast enhanced (DCE) MRI, which involves the serial acquisition of T1-weighted images, before, during, and after the injection of a paramagnetic contrast agent such as gadolinium, provides further insight into the nature of tumor tissue and its close surroundings. DCE imaging reveals characteristics related to tumor vasculature permeability and extravascular extracellular volume (25). DCE imaging can be used to help identify esophageal carcinoma, lymphatic metastases and also predict response to CRT (26-28). Although the performance of DWI and DCE MRI as a single modality are promising, combinations of imaging modalities or 
MRI pulse sequences, may provide complementary value and could further improve the prediction of response to CRT (24, 26, 29).

Similarly, the preSANO trial showed that after nCRT, the use of biopsies, FNA, EUS, in combination with PET-CT could identify $70-90 \%$ of patients with more than $10 \%$ residual carcinoma in the esophagectomy specimen (30). More recently, the prospective PRIDE study aims at the development of a multimodal prediction model including MRI that not only predicts the patients' individual probability of a pCR after nCRT, but also identifies non-responders and patients who are likely to develop distant metastases in the near future (31). Both the SANO and ESOSTRATE trials are comparing active surveillance with immediate surgery in esophageal cancer patients who have achieved a clinical complete response, predicted by PET-CT and endoscopic biopsies, after nCRT $(32,33)$.

\section{RATIONAL FOR MR-GUIDED RADIOTHERAPY IN ESOPHAGEAL CANCER}

Integrated MRI-linear accelerator systems (MR-linacs) provide the ability to adapt the treatment based on daily changes in shape, size and position of the tumor and surrounding tissue in order to increase the accuracy of treatment delivery $(19,20)$. Due to the enhanced soft-tissue contrast, online MRI will allow realtime tumor visualization both before and during beam delivery. In combination with advanced online motion-compensation, MRgRT could well improve tumor targeting accuracy, allow for smaller planning target volume (PTV) margins and consequently result in a reduction of normal tissue exposure with a potential decrease in treatment related toxicity. Moreover, highly accurate tumor targeting with small PTV margins may enable hypofractionation and less toxic dose escalation to eradicative dose levels, potentially omitting the necessity of surgery to control the macroscopic tumor. Daily and even intrafraction plan adaptation and dose painting based on anatomical changes, tumor regression and functional MR imaging will further refine dose escalation and might provide an organ-sparing treatment strategy for a growing number of patients. The potential advantages of MRgRT for esophageal cancer will be discussed below.

\section{Online Interfraction Tumor Shape Adaptation}

The primary tumor, involved nodes and the clinical target volume (CTV) consisting of the peri-esophageal fat often can hardly be discriminated on CBCT. This is particularly true for tumors located in the distal esophagus subject to respiratory and cardiac motion. This is the most common tumor location in the Western world, and often involves the proximal part of the stomach. Hence, set-up corrections are typically performed by online registration of the bony anatomy visible on CBCT, instead of direct matching on the tumor. The interfractional variation of the tumor position and shape in relation to the bony anatomy can be substantial and consequently large PTV margins are required to encompass esophageal tumor (34). Online highquality MRI facilitates online tumor matching, reducing CTV to PTV margins. A recent study has demonstrated that a $10 \mathrm{~mm}$ PTV margin can provide CTV coverage in $89 \%$ of cases where daily set up position is based on a bone match (35). Only a modest improvement in CTV coverage to 93\% could be achieved with a soft tissue, MRI-guided, CTV match with the same $10 \mathrm{~mm}$ margin. This reflects the considerable day-to-day CTV shape changes, especially for distal esophagus and gastroesophageal junction (GEJ), which regularly occurred over the course of treatment and could not be corrected by translational shifts based on soft-tissue registration. This partly explains the modest improvement of geometric coverage of the CTV with online MR-guided soft tissue matching and indicates that correction for the largest interfraction positional variation can only be achieved by daily online adaptation of the target and online replanning (35).

In addition to positional variation of GTV and CTV, substantial tumor volume regression during the course of nCRT can be visualized on MRI. By the fifth week of treatment, esophageal tumors can decrease by $28 \%$ of the initial volume (36). This tumor regression will predominantly result in deformation of the target and, as a consequence, OARs, especially the heart, could move into the initial GTV, thereby increasing the radiation dose to the heart and contributing to cardiac toxicity (Figure 1). The effect of tumor regression on the anatomical configuration can only be appreciated with online MR-guidance and corrected for by an online adaptive workflow where a new treatment plan is generated based on the anatomy of the day. This procedure, also referred to as adapt-to-shape (35) or stereotactic MR-guided adaptive radiation therapy (SMART) (37), will correct for interfraction variation.

\section{Dealing With Intrafraction Tumor Motion}

Intrafraction motion due to respiratory motion revealed by cine MRI average $12-13 \mathrm{~mm}$ in the cranial-caudal (CC) direction, 2.5-5 $\mathrm{mm}$ in the anterior-posterior (AP) direction, and $2.7 \mathrm{~mm}$ in the left-right (LR) direction $(38,39)$. Lower esophageal tumors and GEJ tumors exhibit the largest motion and variability of motion during the respiratory cycle due to their proximity to the diaphragm $(34,40)$. In general, respiratory motion of esophageal tumors will cause a decrease in the sharpness of the dose gradient at the PTV edge, predominantly in CC direction, once the position of the target volume has been properly identified (41). Although the intrafraction motion of esophageal tumors can be categorized as modest and seldom leads to systematic errors, motion management techniques (e.g. respiratory gating, or midposition techniques) are required to bring down CTV-to-PTV margins to 2-3 mm-levels in future treatments. Moreover, drift during treatment can be observed and although in general drifts are small with a mean of $1.5 \mathrm{~mm}$, outliers up to $11 \mathrm{~mm}$ can occur (39).

MRgRT allows for online tumor motion monitoring, which affords the option to intervene in case of extreme anatomical changes and drifts are observed. Moreover, respiratory gating 


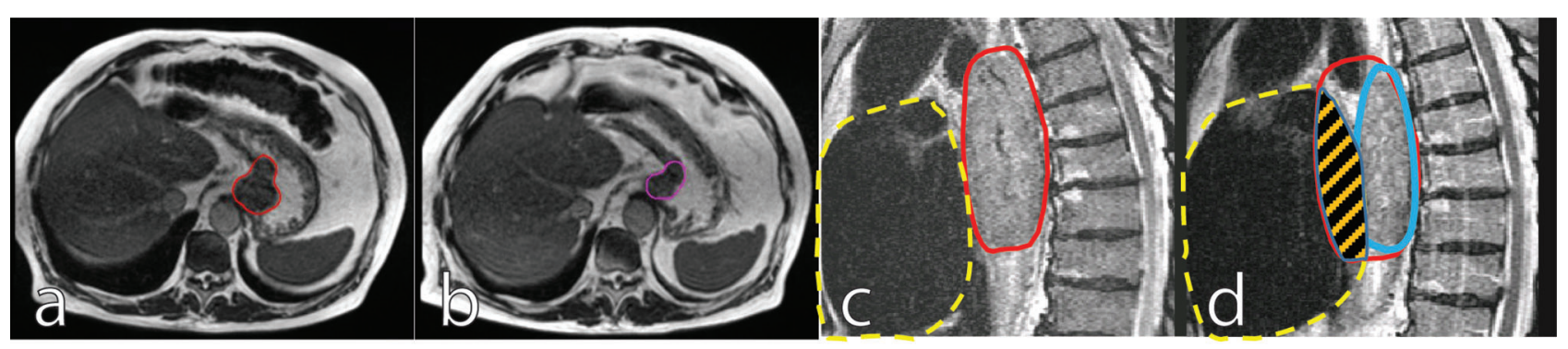

FIGURE 1 | Mid treatment gastroesophageal adenocarcinoma tumor regression: Images are inhale breath hold 0.35T True Fast Imaging with Steady-State Free Precession (TRUFI) at baseline [(A), red outline] and on fraction 10 of chemoradiation therapy [(B), purple outline]. Sagittal views of thoracic squamous cell carcinoma tumor regression depicted 1.5T T2-weighted navigation triggered imaging at baseline [(C), red outline] and on fraction 19 of chemoradiotherapy [(D), blue outline]. In (C, D), the dashed yellow shows the heart contour and the striped orange area shows regression from the overlap of the original tumor volume and the heart volume.

can mitigate the effect of respiratory motion and reduce the required PTV (42). On conventional linear accelerators, respiratory gating is performed using external surrogates, but the correlation between such surrogates and tumor motion can vary substantially (43). As such, image guidance is of utmost importance for accurate respiratory gating to avoid a geographical miss. MRI allows realtime position confirmation during gated treatment by tracking the GTV, ensuring accuracy of the treatment.

\section{Reducing Treatment-Related Toxicity}

Smaller CTV to PTV margins will result in less dose to the surrounding organs at risk and thereby will theoretically decrease treatment related toxicity. In patients undergoing CRT for esophageal cancer, up to $10.8 \%$ develop symptomatic cardiac toxicity (44). Institutional retrospective and database analyses show that compared to patients who undergo esophagectomy alone, those who undergo nCRT have a significantly increased risk of grade 3 or higher cardiac events and that higher radiation doses to the heart correlates with a higher incidence of cardiac events (45-47). In a prospective phase II trial by Lin et al, 145 patients with esophageal cancer were randomized to definitive treatment with proton beam therapy or photon-based intensity-modulated radiation therapy. At a median follow up of 44 months, the total toxicity burden was lower in the proton beam therapy arm, with pronounced numeric differences in cases of atrial fibrillation, asymptomatic effusions, lower-grade pneumonitis, acute respiratory distress syndrome (ARDS) and reintubation. This study demonstrated that the dosimetric advantages of proton therapy resulted in lower rates of toxicity (48). Similar benefits could be expected from daily online adaptive MR-guided radiotherapy plans with tight CTV to PTV margins. MRL treatments using maximum inspiration breath hold under real time MRI tracking can help reduce treatment volumes. In a dosimetric analysis, compared to free breathing treatments on conventional CBCT guided radiotherapy, maximum inspiration breath hold MRL treatments for GEJ tumors can reduce the PTV from $1275 \mathrm{cc}$ to $689 \mathrm{cc}$ with a corresponding decrease in mean heart dose from 27.8 Gy to 20.9 Gy (42). While photon-based MRL treatments may have larger volumes of low dose coverage of OARs, due to uncertainties of the location of the Bragg peak, proton-based treatments are likely to have larger volumes of high dose coverage of proximal OARs. Future studies are warranted to compare the toxicity burdens between photon-based MRL treatments and proton therapy. The ability to visualize moving soft-tissue tumors with MRI and the dosimetric advantages of the Bragg peak with proton therapy could be combined in a hybrid system for MR-integrated proton therapy (MRiPT). Although MRiPT is still in its infancy, research is currently underway to develop prototype systems for clinical use (49). In addition to MRguided daily plan adaptation and PTV margin reduction with consequently better sparing of OARs, MRI may also provide a way to detect subclinical cardiac toxicity after CRT by visualizing areas of myocardial fibrosis and changes in ejection fraction (50).

Besides limiting the radiation dose to the heart, smaller margins with MRgRT can also reduce the dose to the lungs and stomach. Grade 2 or higher radiation pneumonitis affects 5 $7 \%$ of patients undergoing intensity-modulated radiotherapy for esophageal cancer, with greater incidence seen at higher lung V20 doses (51). Recent studies indicate that the ratio of the planning target volume to the total lung volume and the mean lung dose are important for predicting the probability of developing severe acute radiation pneumonitis (52). In patients who undergo esophagectomy, anastomotic leak rates range from $0-24 \%$ and are the cause of $90 \%$ of postoperative mortalities (53). The relationship between nCRT and rates of anastomotic leaks is controversial. The odds ratio for developing an anastomotic leak is 5.37 within the radiation field compared to anastomoses outside the radiation field $(54,55)$. However in studies comparing patients treated with neoadjuvant radiation therapy to resection alone, there was no difference in anastomotic leak rates $(56,57)$. Target definition at the GEJ is challenging and daily variation in this area can be substantial, therefore accurate dose accumulation in the area will be difficult, which might explain the conflicting results.

\section{TARGETED DOSE ESCALATION}

Although progress has been made in the treatment of esophageal cancer, treatment still fails in most patients due to locoregional 
recurrences and the development of metastatic disease. The majority of local recurrences after definitive chemoradiation occurs within the GTV, suggesting a potential benefit of dose escalation in patients unfit for surgery or with unresectable disease $(6,7)$. Furthermore, the generally applied tumor radiation dose of 41.4 to $50.4 \mathrm{~Gy}$ is far below the commonly used doses at other primary tumor sites, such as lung and head and neck tumors. Patients treated with nCRT might benefit from dose escalation by increasing the chance of achieving a pCR. Moreover, patients with a pCR have a favorable prognosis (58) and it could be argued that surgery might be safely omitted in these patients.

Currently, results of dose escalation studies are inconsistent. A landmark randomized trial INT-0123 (RTOG 94-05) revealed that sequential dose escalation to $64.8 \mathrm{~Gy}$ did not translate into an increase in local control or overall survival in esophageal cancer. Radiotherapy techniques have evolved dramatically since the era of the INT-0123 trial and several retrospective and nonrandomized prospective studies have shown an increase in local control after dose escalation (59-61). The ARTDECO trial, published in abstract form in 2020, randomized inoperable esophageal cancer patients (61\% squamous cell carcinoma and $39 \%$ adenocarcinoma) to conventional CRT with a simultaneous integrated boost to a total of $61.6 \mathrm{~Gy}$. Although modern radiation techniques were used, local progression free survival and overall survival were not statistically different between the two groups while the dose escalated arm had higher rates of grade 4-5 toxicity (62). The location and histology of the tumor may influence outcomes of dose escalation studies. Lower esophageal tumors are more challenging to treat. They tend to be adenocarcinoma which are more radioresistant than squamous cell carcinoma, have more cardiac and respiratory motion due to proximity to the heart and diaphragm, are pressed tightly to the adjacent heart, and are limited by proximity and radiosensitivity of the stomach.

The inconsistent results of dose escalation regarding local control and overall survival might also be due to the lack of patient selection. Careful selection of patients for a sequential boost based on the initial PET-CT response to standard CRT showed promising results (63).

\section{FUTURE PROSPECTS OF MRI AND MR- GUIDED RADIOTHERAPY IN ESOPHAGEAL CANCER}

Currently, at the UMC Utrecht the first patients with esophageal cancer are being treated on the 1.5T MR-Linac with reduced margins. Patients receive standard fractionated nCRT with reduced PTV margins (Supplementary Materials, Figure S1). This combined R-Ideal phase 1b-2a study with smaller PTV margins will serve as a proof of concept and the workflow and technology will be further optimized for future innovative treatments, such as dose escalation (64).

MR guided radiotherapy provides an exciting opportunity to improve and personalize esophageal cancer treatment by various means. First, MRI appears to be promising in treatment response assessment to guide patient-tailored treatment strategies, such as dose escalation or organ preservation. Second, online MR guided radiotherapy will result in high precision daily adaptive radiotherapy with reduced margins, thereby reducing toxicity and enabling safe targeted dose escalation. Finally, functional MR-guidance allows for dose painting strategies based on biological information about the tumor in order to increase its efficacy, such as dose escalation to only the parts of the GTV that exhibit persistent tumor activity at the end of standard CRT (Figure 2) (65). Randomized trials are needed to demonstrate the effectiveness of MR-guided radiotherapy compared to conventional CBCT guided radiotherapy.

In addition, the increased accuracy of online MR guided radiotherapy, due to daily adaptation of target delineation and online replanning in combination with beam on imaging, might pave the way for hypofractionated dose escalation in esophageal cancer. Hypofractionated radiotherapy has the advantage of a shorter overall treatment time and a higher biological

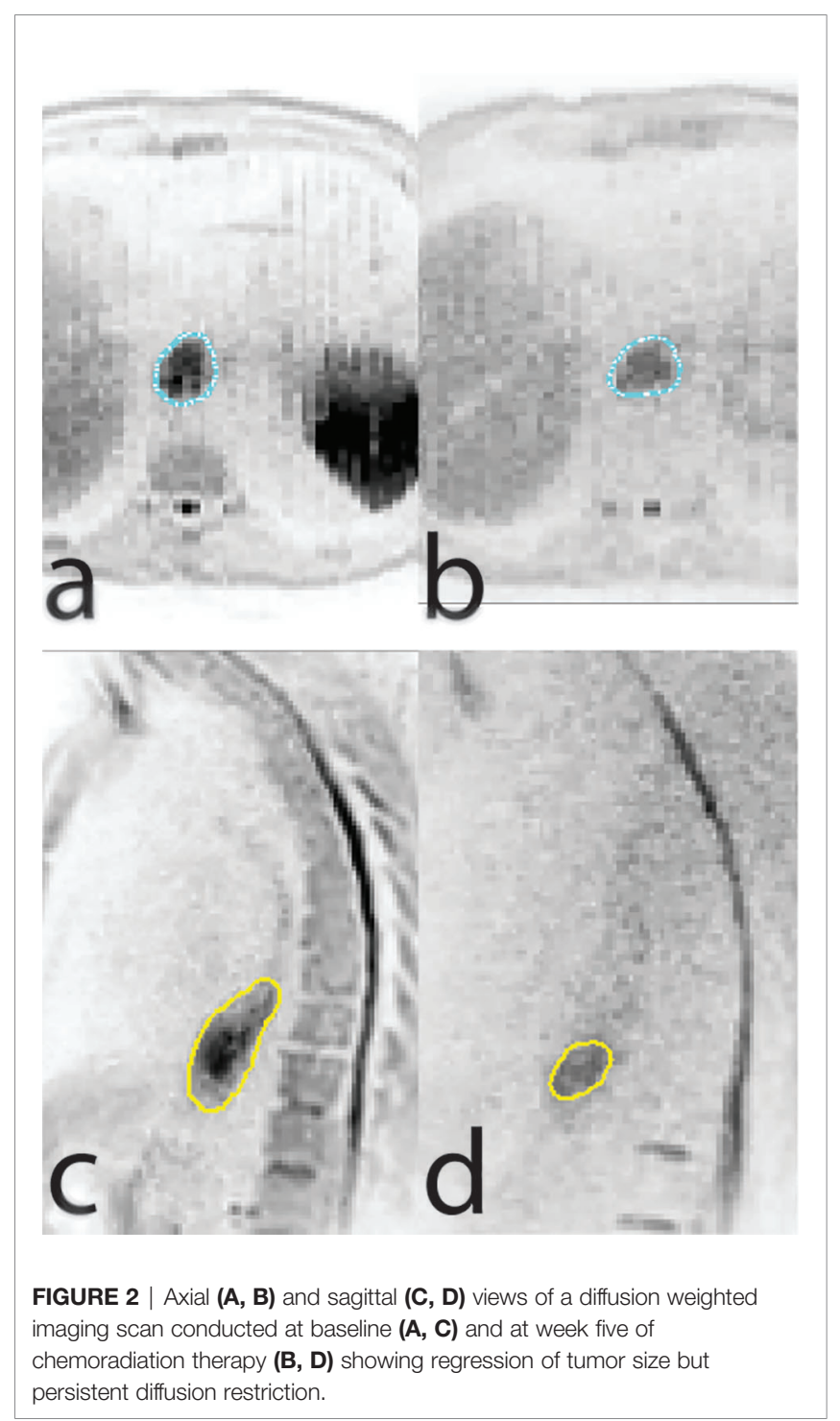


effectiveness. Future studies need to elucidate whether hypofractionated radiotherapy will improve outcomes in esophageal cancer in terms of local control, will lead to adequate functional outcomes and is safe in terms of esophageal toxicity.

\section{DATA AVAILABILITY STATEMENT}

The original contributions presented in the study are included in the article/Supplementary Material. Further inquiries can be directed to the corresponding author.

\section{REFERENCES}

1. Bray F, Ferlay J, Soerjomataram I, Siegel RL, Torre LA, Jemal A. Global cancer statistics 2018: GLOBOCAN estimates of incidence and mortality worldwide for 36 cancers in 185 countries. CA: A Cancer J Clin (2018) 68:394-424. doi: $10.3322 /$ caac. 21492

2. Sjoquist KM, Burmeister BH, Smithers BM, Zalcberg JR, Simes RJ, Barbour A, et al. Survival after neoadjuvant chemotherapy or chemoradiotherapy for resectable oesophageal carcinoma: an updated meta-analysis. Lancet Oncol (2011) 12:681-92. doi: 10.1016/S1470-2045(11)70142-5

3. Shapiro J, van Lanschot JJB, Hulshof MCCM, van Hagen P, van Berge Henegouwen MI, Wijnhoven BPL, et al. Neoadjuvant chemoradiotherapy plus surgery versus surgery alone for oesophageal or junctional cancer (CROSS): long-term results of a randomised controlled trial. Lancet Oncol (2015) 16:1090-8 doi: 10.1016/S1470-2045(15)00040-6.

4. Teoh AYB, Chiu PWY, Yeung WK, Liu SYW, Wong SKH, Ng EKW. Longterm survival outcomes after definitive chemoradiation versus surgery in patients with resectable squamous carcinoma of the esophagus: results from a randomized controlled trial. Ann Oncol (2013) 24:165-71. doi: 10.1093/ annonc/mds206

5. Gwynne S, Hurt C, Evans M, Holden C, Vout L, Crosby T. Definitive Chemoradiation for Oesophageal Cancer - a Standard of Care in Patients with Non-metastatic Oesophageal Cancer. Clin Oncol (2011) 23:182-8. doi: 10.1016/j.clon.2010.12.001

6. Welsh J, Settle SH, Amini A, Xiao L, Suzuki A, Hayashi Y, et al. Failure patterns in patients with esophageal cancer treated with definitive chemoradiation: Failure After Esophageal Chemoradiation. Cancer (2012) 118:2632-40. doi: 10.1002/cncr.26586

7. Versteijne E, van Laarhoven HWM, van Hooft JE, van Os RM, Geijsen ED, van Berge Henegouwen MI, et al. Definitive chemoradiation for patients with inoperable and/or unresectable esophageal cancer: locoregional recurrence pattern: dCRT oesophageal cancer: recurrence pattern. Dis Esophagus (2015) 28:453-9. doi: 10.1111/dote.12215

8. Encinas de la Iglesia J, Corral de la Calle MA, Fernández Pérez GC, Ruano Pérez R, Álvarez Delgado A. Esophageal cancer: Anatomic particularities, staging, and imaging techniques. Radiol (English Ed) (2016) 58:352-65. doi: 10.1016/j.rxeng.2016.06.002

9. van Rossum PSN, van Hillegersberg R, Lever FM, Lips IM, van Lier ALHMW, Meijer GJ, et al. Imaging strategies in the management of oesophageal cancer: what's the role of MRI? Eur Radiol (2013) 23:1753-65. doi: 10.1007/s00330013-2773-6

10. Riddell AM, Richardson C, Scurr E, Brown G. The development and optimization of high spatial resolution MRI for imaging the oesophagus using an external surface coil. BJR (2006) 79:873-9. doi: 10.1259/bjr/36989440

11. Riddell AM, Hillier J, Brown G, King DM, Wotherspoon AC, Thompson JN, et al. Potential of Surface-Coil MRI for Staging of Esophageal Cancer. Am J Roentgenol (2006) 187:1280-7. doi: 10.2214/AJR.05.0559

12. Vollenbrock SE, Nowee ME, Voncken FEM, Kotte ANTJ, Goense L, van Rossum PSN, et al. Gross Tumor Delineation in Esophageal Cancer on MRI Compared With 18F-FDG-PET/CT. Adv Radiat Oncol (2019) 4:596-604. doi: 10.1016/j.adro.2019.04.004

\section{AUTHOR CONTRIBUTIONS}

$\mathrm{SL}, \mathrm{MB}$, and SM were responsible for the conception of this review. All authors contributed to the article and approved the submitted version.

\section{SUPPLEMENTARY MATERIAL}

The Supplementary Material for this article can be found online at: https://www.frontiersin.org/articles/10.3389/fonc.2021. 628009/full\#supplementary-material

13. Hou D-L, Shi G-F, Gao X-S, Asaumi J, Li X-Y, Liu H, et al. Improved longitudinal length accuracy of gross tumor volume delineation with diffusion weighted magnetic resonance imaging for esophageal squamous cell carcinoma. Radiat Oncol (2013) 8:169. doi: 10.1186/1748-717X-8-169

14. van Hagen P, Hulshof MCCM, van Lanschot JJB, Steyerberg EW, van Berge Henegouwen MI, Wijnhoven BPL, et al. Preoperative chemoradiotherapy for esophageal or junctional cancer. N Engl J Med (2012) 366:2074-84. doi: 10.1056/NEJMoa1112088

15. Scarpa M, Valente S, Alfieri R, Cagol M, Diamantis G, Ancona E, et al. Systematic review of health-related quality of life after esophagectomy for esophageal cancer. World J Gastroenterol (2011) 17:4660-74. doi: 10.3748/ wjg.v17.i42.4660

16. Eyck BM, Onstenk BD, Noordman BJ, Nieboer D, Spaander MCW, Valkema $\mathrm{R}$, et al. Accuracy of Detecting Residual Disease After Neoadjuvant Chemoradiotherapy for Esophageal Cancer: A Systematic Review and Metaanalysis. Ann Surg (2020) 271:245-56. doi: 10.1097/SLA.0000000000003397

17. de Gouw DJJM, Klarenbeek BR, Driessen M, Bouwense SAW, van Workum F, Fütterer JJ, et al. Detecting Pathological Complete Response in Esophageal Cancer after Neoadjuvant Therapy Based on Imaging Techniques: A Diagnostic Systematic Review and Meta-Analysis. J Thoracic Oncol (2019) 14:1156-71. doi: 10.1016/j.jtho.2019.04.004

18. Leibfarth S, Winter RM, Lyng H, Zips D, Thorwarth D. Potentials and challenges of diffusion-weighted magnetic resonance imaging in radiotherapy. Clin Trans Radiat Oncol (2018) 13:29-37. doi: 10.1016/ j.ctro.2018.09.002

19. van Rossum PSN, van Lier ALHMW, van Vulpen M, Reerink O, Lagendijk JJW, Lin SH, et al. Diffusion-weighted magnetic resonance imaging for the prediction of pathologic response to neoadjuvant chemoradiotherapy in esophageal cancer. Radiother Oncol (2015) 115:163-70. doi: 10.1016/ j.radonc.2015.04.027

20. Borggreve AS, Heethuis SE, Boekhoff MR, Goense L, van Rossum PSN, Brosens LAA, et al. Optimal timing for prediction of pathologic complete response to neoadjuvant chemoradiotherapy with diffusion-weighted MRI in patients with esophageal cancer. Eur Radiol (2020) 30:1896-907. doi: 10.1007/ s00330-019-06513-0

21. Aoyagi T, Shuto K, Okazumi S, Shimada H, Kazama T, Matsubara H. Apparent Diffusion Coefficient Values Measured by Diffusion-Weighted Imaging Predict Chemoradiotherapeutic Effect for Advanced Esophageal Cancer. Dig Surg (2011) 28:252-7. doi: 10.1159/000328770

22. Li FP, Wang H, Hou J, Tang J, Lu Q, Wang LL, et al. Utility of intravoxel incoherent motion diffusion-weighted imaging in predicting early response to concurrent chemoradiotherapy in oesophageal squamous cell carcinoma. Clin Radiol (2018) 73:756.e17-26. doi: 10.1016/j.crad.2018.03.015

23. Imanishi S, Shuto K, Aoyagi T, Kono T, Saito H, Matsubara H. DiffusionWeighted Magnetic Resonance Imaging for Predicting and Detecting the Early Response to Chemoradiotherapy of Advanced Esophageal Squamous Cell Carcinoma. DSU (2013) 30:240-8. doi: 10.1159/000351435

24. Fang P, Musall BC, Son JB, Moreno AC, Hobbs BP, Carter BW, et al. Multimodal Imaging of Pathologic Response to Chemoradiation in Esophageal Cancer. Int J Radiat Oncol Biol Physics (2018) 102:996-1001. doi: 10.1016/j.ijrobp.2018.02.029 
25. Barnes SL, Whisenant JG, Li X, Yankeelov TE. Techniques and applications of dynamic contrast enhanced magnetic resonance imaging in cancer. In: 2014 36th Annual International Conference of the IEEE Engineering in Medicine and Biology Society (2014). p. 4264-7. doi: 10.1109/EMBC.2014.6944566

26. Heethuis SE, Goense L, van Rossum PSN, Borggreve AS, Mook S, Voncken FEM, et al. DW-MRI and DCE-MRI are of complementary value in predicting pathologic response to neoadjuvant chemoradiotherapy for esophageal cancer. Acta Oncol (2018) 57:1201-8. doi: 10.1080/0284186X.2018.1473637

27. Lei J, Han Q, Zhu S, Shi D, Dou S, Su Z, et al. Assessment of esophageal carcinoma undergoing concurrent chemoradiotherapy with quantitative dynamic contrast-enhanced magnetic resonance imaging. Oncol Lett (2015) 10:3607-12. doi: 10.3892/ol.2015.3779

28. Chen Y, Jiang Y, Chen T, Li R, Zhang X, Chen F, et al. Assessing Microcirculation in Resectable Oesophageal Squamous Cell Carcinoma with Dynamic Contrast-enhanced MRI for Identifying Primary tumour and Lymphatic Metastasis. Sci Rep (2019) 9:124. doi: 10.1038/s41598-01836929-5

29. Borggreve AS, Goense L, van Rossum PSN, Heethuis SE, van Hillegersberg R, Lagendijk JJW, et al. Preoperative Prediction of Pathologic Response to Neoadjuvant Chemoradiotherapy in Patients With Esophageal Cancer Using 18F-FDG PET/CT and DW-MRI: A Prospective Multicenter Study. Int J Radiat Oncol Biol Physics (2020) 106:998-1009. doi: 10.1016/ j.ijrobp.2019.12.038

30. Noordman BJ, Spaander MCW, Valkema R, Wijnhoven BPL, van Berge Henegouwen MI, Shapiro J, et al. Detection of residual disease after neoadjuvant chemoradiotherapy for oesophageal cancer (preSANO): a prospective multicentre, diagnostic cohort study. Lancet Oncol (2018) 19:965-74. doi: 10.1016/S1470-2045 (18)30201-8.

31. on behalf of the PRIDE study group, Borggreve AS, Mook S, Verheij M, Mul VEM, Bergman JJ, et al. Preoperative image-guided identification of response to neoadjuvant chemoradiotherapy in esophageal cancer (PRIDE): a multicenter observational study. BMC Cancer (2018) 18:1006. doi: 10.1186/ s12885-018-4892-6

32. on behalf of the SANO-study group, Noordman BJ, Wijnhoven BPL, Lagarde SM, Boonstra JJ, Coene PPLO, et al. Neoadjuvant chemoradiotherapy plus surgery versus active surveillance for oesophageal cancer: a stepped-wedge cluster randomised trial. BMC Cancer (2018) 18:142. doi: 10.1186/s12885018-4034-1

33. Donohoe CL, Reynolds JV. Neoadjuvant treatment of locally advanced esophageal and junctional cancer: the evidence-base, current key questions and clinical trials. J Thorac Dis. (2017) 9. doi: 10.21037/jtd.2017.03.159

34. Jin P, Hulshof MCCM, van Wieringen N, Bel A, Alderliesten T. Interfractional variability of respiration-induced esophageal tumor motion quantified using fiducial markers and four-dimensional cone-beam computed tomography. Radiother Oncol (2017) 124:147-54. doi: 10.1016/j.radonc.2017.05.015

35. Winkel D, Bol GH, Kroon PS, van Asselen B, Hackett SS, WerensteijnHoningh AM, et al. Adaptive radiotherapy: The Elekta Unity MR-linac concept. Clin Trans Radiat Oncol (2019) 18:54-9. doi: 10.1016/ j.ctro.2019.04.001

36. Defize IL, Boekhoff MR, Borggreve AS, van Lier ALHMW, Takahashi N, Haj Mohammad $\mathrm{N}$, et al. Tumor volume regression during neoadjuvant chemoradiotherapy for esophageal cancer: a prospective study with weekly MRI. Acta Oncol (2020) 59:753-9. doi: 10.1080/0284186X.2020.1759819

37. Bohoudi O, Bruynzeel AME, Senan S, Cuijpers JP, Slotman BJ, Lagerwaard FJ, et al. Fast and robust online adaptive planning in stereotactic MR-guided adaptive radiation therapy (SMART) for pancreatic cancer. Radiother Oncol (2017) 125(3):439-44. doi: 10.1016/j.radonc.2017.07.028

38. Lever FM, Lips IM, Crijns SPM, Reerink O, van Lier ALHMW, Moerland MA, et al. Quantification of Esophageal Tumor Motion on Cine-Magnetic Resonance Imaging. Int J Radiat Oncol Biol Physics (2014) 88:419-24. doi: 10.1016/j.ijrobp.2013.10.036

39. Heethuis SE, Borggreve AS, Goense L, van Rossum PSN, Mook S, van Hillegersberg R, et al. Quantification of variations in intra-fraction motion of esophageal tumors over the course of neoadjuvant chemoradiotherapy based on cine-MRI. Phys Med Biol (2018) 63:145019. doi: 10.1088/1361-6560/ aacfb5

40. Zhou H-Y, Zhang J-G, Li R, Zhang X-M, Chen T-W, Liu N, et al. Tumour motion of oesophageal squamous cell carcinoma evaluated by cine MRI: associated with tumour location. Clin Radiol (2018) 73:676.e1-7. doi: 10.1016/ j.crad.2018.02.006

41. Boekhoff MR, Defize IL, Borggreve AS, Takahashi N, van Lier ALHMW, Ruurda JP, et al. 3-Dimensional target coverage assessment for MRI guided esophageal cancer radiotherapy. Radiother Oncol (2020) 147:1-7. doi: 10.1016/j.radonc.2020.03.007

42. Lee SL, Mahler P, Olson S, Witt JS, Musunuru HB, Rajamanickam V, et al. Reduction of cardiac dose using respiratory-gated MR-linac plans for gastroesophageal junction cancer. Med Dosimetry (2020) 2020:S0958394720301382. doi: 10.1016/j.meddos.2020.10.002

43. Voncken FEM, Nakhaee S, Stam B, Wiersema L, Vollenbrock SE, van Dieren JM, et al. Quantification of Esophageal Tumor Motion and Investigation of Different Image-Guided Correction Strategies. Pract Radiat Oncol (2020) 10:84-92. doi: 10.1016/j.prro.2019.11.012

44. Beukema JC, van Luijk P, Widder J, Langendijk JA, Muijs CT. Is cardiac toxicity a relevant issue in the radiation treatment of esophageal cancer? Radiother Oncol (2015) 114:85-90. doi: 10.1016/j.radonc.2014.11.037

45. Hayashi Y, Iijima H, Isohashi F, Tsujii Y, Fujinaga T, Nagai K, et al. The heart's exposure to radiation increases the risk of cardiac toxicity after chemoradiotherapy for superficial esophageal cancer: a retrospective cohort study. BMC Cancer (2019) 19:1-7. doi: 10.1186/s12885-019-5421-y

46. Witt JS, Jagodinsky JC, Liu Y, Yadav P, Kuczmarska-Haas A, Yu M, et al. Cardiac Toxicity in Operable Esophageal Cancer Patients Treated With or Without Chemoradiation. Am J Clin Oncol (2019) 42:662-7. doi: 10.1097/ COC.0000000000000573

47. Gharzai L, Verma V, Denniston KA, Bhirud AR, Bennion NR, Lin C. Radiation Therapy and Cardiac Death in Long-Term Survivors of Esophageal Cancer: An Analysis of the Surveillance, Epidemiology, and End Result Database. PloS One (2016) 11:e0158916. doi: 10.1371/ journal.pone.0158916

48. Lin SH, Hobbs BP, Verma V, Tidwell RS, Smith GL, Lei X, et al. Randomized Phase IIB Trial of Proton Beam Therapy Versus Intensity-Modulated Radiation Therapy for Locally Advanced Esophageal Cancer. JCO (2020) 2020. doi: 10.1200/JCO.19.02503

49. Hoffmann A, Oborn B, Moteabbed M, Yan S, Bortfeld T, Knopf A, et al. MRguided proton therapy: a review and a preview. Radiat Oncol (2020) 15:129. doi: 10.1186/s13014-020-01571-x

50. Burke AM, Yeh C, Kim S, Bergquist P, Krishnan P, Barac A, et al. A Prospective Study of Early Radiation Associated Cardiac Toxicity Following Neoadjuvant Chemoradiation for Distal Esophageal Cancer. Front Oncol (2020) 10:1169. doi: 10.3389/fonc.2020.01169

51. Tonison JJ, Fischer SG, Viehrig M, Welz S, Boeke S, Zwirner K, et al. Radiation Pneumonitis after Intensity-Modulated Radiotherapy for Esophageal Cancer: Institutional Data and a Systematic Review. Sci Rep (2019) 9:2255. doi: 10.1038/s41598-018-38414-5

52. Wang L, Liang S, Li C, Sun X, Pang L, Meng X, et al. A Novel Nomogram and Risk Classification System Predicting Radiation Pneumonitis in Patients With Esophageal Cancer Receiving Radiation Therapy. Int J Radiat Oncol Biol Physics (2019) 105:1074-85. doi: 10.1016/j.ijrobp.2019.08.024

53. Kim RH, Takabe K. Methods of esophagogastric anastomoses following esophagectomy for cancer: A systematic review. J Surg Oncol (2010) 101:527-33. doi: 10.1002/jso. 21510

54. Juloori A, Tucker SL, Komaki R, Liao Z, Correa AM, Swisher SG, et al. Influence of Preoperative Radiation Field on Postoperative Leak Rates in Esophageal Cancer Patients after Trimodality Therapy. J Thoracic Oncol (2014) 9:534-40. doi: 10.1097/JTO.0000000000000100

55. Goense L, van Rossum PSN, Tromp M, Joore HC, van Dijk D, Kroese AC, et al. Intraoperative and postoperative risk factors for anastomotic leakage and pneumonia after esophagectomy for cancer. Dis Esophagus (2017) 30:1-10. doi: 10.1111 /dote. 12517

56. Koëter M, van der Sangen MJ, Hurkmans CW, Luyer MD, Rutten HJ, Nieuwenhuijzen GA, et al. Radiation dose does not influence anastomotic complications in patients with esophageal cancer treated with neoadjuvant chemoradiation and transhiatal esophagectomy. Radiat Oncol (2015) 10:1-11. doi: 10.1186/s13014-015-0361-4

57. Shridhar R, Takahashi C, Huston J, Doepker MP, Meredith KL. Anastomotic leak and neoadjuvant chemoradiotherapy in esophageal cancer. J Gastrointestinal Oncol (2018) 9:894-902. doi: 10.21037/jgo.2018.04.09 
58. Groth SS, Burt BM, Farjah F, Smaglo BG, Sada YH, Sugarbaker DJ, et al. Prognostic value of neoadjuvant treatment response in locally advanced esophageal adenocarcinoma. J Thoracic Cardiovasc Surg (2019) 157:168293.e1. doi: 10.1016/j.jtcvs.2018.11.131

59. Chang C-L, Tsai H-C, Lin W-C, Chang J-H, Hsu H-L, Chow J-M, et al. Dose escalation intensity-modulated radiotherapy-based concurrent chemoradiotherapy is effective for advanced-stage thoracic esophageal squamous cell carcinoma. Radiother Oncol (2017) 125:73-9. doi: 10.1016/ j.radonc.2017.08.025

60. Chen D, Menon H, Verma V, Seyedin SN, Ajani JA, Hofstetter WL, et al. Results of a Phase 1/2 Trial of Chemoradiotherapy With Simultaneous Integrated Boost of Radiotherapy Dose in Unresectable Locally Advanced Esophageal Cancer. JAMA Oncol (2019) 5:1597. doi: 10.1001/ jamaoncol.2019.2809

61. Zhang W, Luo Y, Wang X, Han G, Wang P, Yuan W, et al. Dose-escalated radiotherapy improved survival for esophageal cancer patients with a clinical complete response after standard-dose radiotherapy with concurrent chemotherapy. CMAR (2018) 10:2675-82. doi: 10.2147/CMAR.S160909

62. Hulshof MCCM, Geijsen D, Rozema T, Oppedijk V, Buijsen J, Neelis KJ, et al. A randomized controlled phase III multicenter study on dose escalation in definitive chemoradiation for patients with locally advanced esophageal cancer: ARTDECO study. JCO (2020) 38:281.

63. Ma J, Wang Z, Wang C, Chen E, Dong Y, Song Y, et al. Individualized Radiation Dose Escalation Based on the Decrease in Tumor FDG Uptake and Normal Tissue Constraints Improve Survival in Patients With Esophageal
Carcinoma. Technol Cancer Res Treat (2017) 16:75-80. doi: 10.1177/ 1533034615627583

64. Verkooijen HM, Kerkmeijer LGW, Fuller CD, Huddart R, Faivre-Finn C, Verheij M, et al. R-IDEAL: A Framework for Systematic Clinical Evaluation of Technical Innovations in Radiation Oncology. Front Oncol (2017) 7:64. doi: 10.3389/fonc.2017.00059

65. Yu W, Cai X-W, Liu Q, Zhu Z-F, Feng W, Zhang Q, et al. Safety of dose escalation by simultaneous integrated boosting radiation dose within the primary tumor guided by 18FDG-PET/CT for esophageal cancer. Radiother Oncol (2015) 114:195-200. doi: 10.1016/j.radonc.2014.12.007

Conflict of Interest: MB reports non-financial support from Viewray Inc, outside the submitted work, and MERCK Clinical Trial Support, Astra Zeneca Clinical Trial Support, EMD Serono Clinical Trial Support. SL reports non-financial support from ViewRay, outside the submitted work. GM and SM are both members of the international MR-linac Consortium, which is financially supported by Elekta. The UMC Utrecht Department of Radiotherapy is financially supported by Elekta.

Copyright (c) 2021 Lee, Bassetti, Meijer and Mook. This is an open-access article distributed under the terms of the Creative Commons Attribution License (CC BY). The use, distribution or reproduction in other forums is permitted, provided the original author(s) and the copyright owner(s) are credited and that the original publication in this journal is cited, in accordance with accepted academic practice. No use, distribution or reproduction is permitted which does not comply with these terms. 